\title{
Entrenamiento de la flexibilidad en voleibol sentado*
}

\author{
Gil Andersson Cantillo Bello** \\ Diana Alexandra Camargo Rojas ${ }^{* * *}$
}

Recibido: julio 5 de 2019 • Aceptado: septiembre 25 de 2019

\section{Resumen}

La presente investigación es un estudio cuasiexperimental que muestra los efectos de un programa de entrenamiento de la flexibilidad de la cadena muscular posterior sobre el alcance funcional y la ejecución del golpe de antebrazos en un grupo de jugadores de voleibol sentado de la liga de Bogotá. Dicho programa se fundamentó en la combinación de dos técnicas de desarrollo de la flexibilidad: facilitación neuromuscular propioceptiva (FNP) y estiramiento dinámico. Se llevó a cabo durante seis semanas, para un total de 17 sesiones, cada una de 30 minutos de duración, y dos sesiones para la aplicación del pretest y el postest. El estudio mostró una significancia estadística en los resultados de los test de flexibilidad; sin embargo, no se obtuvo significancia en los resultados del test de alcance funcional, ni en la evaluación del golpe de antebrazos, aunque sí se observaron cambios a nivel

\footnotetext{
"Artículo de investigación, con financiación propia. Grupo de investigación en Actividad Física y Motricidad Humana de la Corporación Universitaria CENDA, Bogotá, Colombia. Citar como: Cantillo, A. y Camargo, D. (2019). Entrenamiento de la flexibilidad en voleibol sentado. Revista de Investigación Cuerpo, cultura y movimiento, 9(2), 103-119. DoI: https:// doi.org/10.15332/2422474x/5363

"* Especialista en Discapacidad, Educación Física, Recreación y Deporte Adaptados de la Corporación Universitaria CENDA, Bogotá, Colombia. Correo electrónico: andertm@gmail.com / ORCID: https://orcid.org/0000-0002-7102-9498

**** Magíster en Salud Pública, profesora de la Corporación Universitaria CENDA, Bogotá, Colombia; profesora de la Universidad Nacional de Colombia, Bogotá, Colombia. Correo electrónico: dacamargor@unal.edu.co / oRcID: https://orcid.org/0000-0003-0922-1603
} 
clínico. Además, se encontró una correlación estadísticamente significativa entre el test sit-and-reach y el ángulo de inclinación del tronco, que fue medido a través del análisis de gesto en el software Kinovea ${ }^{\circledR}$.

Palabras clave: flexibilidad de la cadena posterior, alcance funcional, golpe de antebrazos, voleibol sentado. 


\title{
Flexibility training in sitting volleyball
}

\begin{abstract}
The present investigation is a quasi-experimental study that shows the effects of a training program of the flexibility of the posterior chain on the functional reach and execution of the forearm pass in a group of sitting volleyball players from the Bogota league. This program was based on the combination of two techniques of flexibility development: proprioceptive neuromuscular facilitation (PNF) and dynamic stretching. It lasted six weeks, 17 sessions, each session of 30 minutes and two sessions for applying the pretest and posttest. The study shows statistical significance in the results of the flexibility test; however the significance in the results of the functional reach test or in the evaluation of the forearm pass is not obtained, although changes at clinical level are observed. There is also a correlation of statistical significance between the sit and reach test and the trunk inclination angle, measured through gesture analysis using Kinovea ${ }^{\circledR}$ software.
\end{abstract}

Keywords: : flexibility of the posterior chain, functional reach, forearm pass, sitting volleyball. 


\section{Introducción}

El voleibol sentado es un deporte adaptado del voleibol convencional, por este motivo comparte la mayoría de sus principios técnicos y dinámicas de juego, con algunas variaciones o adaptaciones. Como lo describe Sanz (2013-2014), estas son realizadas con el fin (como en todos los deportes adaptados) de permitir la participación de personas con discapacidad física. El voleibol sentado es un deporte perteneciente al grupo de deportes paralímpicos. En Colombia ha tenido un gran desarrollo e impacto social (Ruiz, 2012), debido a que gran parte de los deportistas que lo practican han sido víctimas del conflicto armado, como lo afirman Muñoz y Montes (2017), en su investigación, cuyo objetivo era interpretar el sentido que el voleibol sentado tiene en los practicantes de este deporte en Colombia.

Por otro lado, es importante mencionar que algunas de las adaptaciones que se realizan en el voleibol sentado son: a) la disminución del tamaño (área) de la cancha, b) la disminución de la altura de la malla, c) se reglamenta que los jugadores deben permanecer en posición sedente con los glúteos en contacto con el suelo, entre otras (World ParaVolley, 2017). Esta última regla exige mayor flexibilidad para participar en las diferentes acciones de juego, principalmente en aquellas en las que el jugador requiere inclinar su cuerpo hacia al frente para tener contacto con el balón, como en el golpe de antebrazos.

Para la ejecución del golpe de antebrazos, Castro (2010) afirma que "la cadena compuesta por brazos-hombros-espalda, cumple la función del movimiento de piernas" (p. 1). El jugador requiere flexionar el tronco hacia el frente; este movimiento tiene un componente importante de flexibilidad de la cadena muscular posterior. Por tal razón, el presente proyecto plantea que si la flexibilidad de la cadena muscular posterior mejora, el alcance funcional modificado (máxima distancia que se puede recorrer hacia el frente en posición sedente, realizando inclinación del tronco) y la ejecución del golpe de antebrazos del jugador de voleibol sentado podrían mejorar. Esta relación de las cadenas musculares en el desarrollo de la flexibilidad y los movimientos de flexión-extensión de tronco ha sido conceptualizada. Al respecto, se destaca el planteamiento de Busquet (1994), "la flexión y extensión del tronco depende de las cadenas rectas, se efectúan con relación a dos ejes miotensivos importantes, uno anterior y uno posterior” (p. 29). 
En relación con las investigaciones realizadas en Colombia en voleibol sentado, se encuentran dos referentes teóricos. El primero publicado por Herrera, Ordóñez, Posada y Landinez (2014), quienes buscaron determinar los cambios en el balance estático y dinámico en jugadores de voleibol sentado de la selección Colombia masculina, después del entrenamiento de balance basado en Biofeedback-совs. El estudio concluyó que, aunque se evidenciaron cambios en el grupo intervención con respecto al control, no hubo significancia estadística. El segundo estudio, realizado por Guerrero y Garnica (2018), examinó los efectos del entrenamiento de fuerza y flexibilidad en el alcance vertical en los jugadores de la selección Bogotá de voleibol sentado. Este concluyó no haber encontrado una mejora significativa en el alcance vertical luego de la implementación del entrenamiento de fuerza y flexibilidad. A partir de estas investigaciones, se puede observar que el concepto de alcance funcional en el voleibol sentado no se encuentra documentado, así como tampoco el efecto del entrenamiento de la flexibilidad sobre el alcance funcional y el golpe de antebrazos.

Con base en los planteamientos anteriores, surge la necesidad de determinar qué efectos tiene la implementación de un programa de entrenamiento de la flexibilidad de la cadena muscular posterior sobre el alcance funcional y la ejecución del golpe de antebrazos en voleibol sentado, entendiendo que, como lo manifiesta Marszalek y colaboradores (2015), es necesario buscar las diferentes relaciones entre los diversos factores que afectan el rendimiento deportivo en las personas con discapacidad.

\section{Metodología}

El desarrollo del presente estudio hace uso de un corpus de conocimientos científico-deportivos, los cuales, a la vez, pertenecen a las ciencias aplicadas del deporte y la actividad física (Arias, 2011, p. 5). Se utilizó la metodología de un estudio cuasiexperimental, el cual empleó “[...] la recolección de datos para probar hipótesis, con base en la medición numérica y el análisis estadístico, para establecer patrones de comportamiento y probar teorías" (Hernández, Fernández y Baptista, 2010, p. 4). Este estudio se desarrolló en la selección masculina de voleibol sentado de la Liga de Bogotá (tabla 1). 
Tabla 1. Variables de la investigación

\begin{tabular}{|c|c|c|c|c|}
\hline Variable & Definición & Unidad & Instrumento & Tipo \\
\hline $\begin{array}{c}\text { Flexibilidad de la } \\
\text { cadena muscular } \\
\text { posterior, Sit-and- } \\
\text { Reach (Sainz, Ayala, } \\
\text { Cejudo y Santonja, } \\
2012\end{array}$ & $\begin{array}{l}\text { Es la capacidad de } \\
\text { alcanzar grandes } \\
\text { excursiones articulares } \\
\text { en los movimientos que } \\
\text { implican extensión de la } \\
\text { cadena muscular posterior. }\end{array}$ & $\mathrm{Cm}$ & $\begin{array}{l}\text { Cajón para test } \\
\text { sit-and-reach }\end{array}$ & I \\
\hline $\begin{array}{l}\text { Alcance funcional } \\
\text { modificado }\end{array}$ & $\begin{array}{l}\text { Máxima distancia que se } \\
\text { puede recorrer hacia el } \\
\text { frente en posición sedente, } \\
\text { realizando inclinación del } \\
\text { tronco. }\end{array}$ & $\mathrm{Cm}$ & $\begin{array}{l}\text { Cinta métrica y } \\
\text { silla con espaldar } \\
\text { preferiblemente } \\
\text { ajustable }\end{array}$ & $\mathrm{D}$ \\
\hline Golpe de antebrazos & $\begin{array}{l}\text { Gesto técnico del voleibol } \\
\text { sentado, en el cual el } \\
\text { jugador golpea el balón } \\
\text { usando los antebrazos y } \\
\text { buscando una trayectoria } \\
\text { parabólica que facilite la } \\
\text { consecución de la jugada. }\end{array}$ & $\begin{array}{l}\text { Ángulos- } \\
\text { grados }\end{array}$ & $\begin{array}{c}\text { Software Kinovea } \\
\text { y marcadores para } \\
\text { puntos } \\
\text { antropométricos. } \\
\text { Cámara de video y } \\
\text { trípodes. }\end{array}$ & $\mathrm{D}$ \\
\hline $\begin{array}{l}\text { Flexibilidad y } \\
\text { movilidad de } \\
\text { hombro (shoulder } \\
\text { streetch y sholder } \\
\text { neck mobility) }\end{array}$ & $\begin{array}{l}\text { Es la capacidad de } \\
\text { alcanzar grandes } \\
\text { excursiones articulares } \\
\text { en la articulación del } \\
\text { hombro. }\end{array}$ & $\begin{array}{l}\text { A percepción } \\
\text { del evaluador } \\
\text { bajo escalas } \\
\text { estandarizadas }\end{array}$ & Cinta métrica & $\mathrm{I} 2$ \\
\hline $\begin{array}{l}\text { Fuerza lumbodorsal } \\
\text { y flexibilidad del } \\
\text { tronco en extensión, } \\
\text { trunk lift (Morales, } \\
\text { 2015) }\end{array}$ & $\begin{array}{l}\text { Fuerza y flexibilidad de } \\
\text { la musculatura lumbar } \\
\text { y dorsal, la cual permite } \\
\text { realizar ejercicios de } \\
\text { extensión del tronco. }\end{array}$ & $\mathrm{Cm}$ & Cinta métrica & $\mathrm{I} 2$ \\
\hline
\end{tabular}

Fuente: elaboración propia.

Las pruebas descritas en la tabla anterior son estandarizadas y de conocimiento en el campo del deporte. En el caso de la evaluación de la ejecución golpe de antebrazos, se emplea el análisis de gesto deportivo, luego de considerar que este permite observar la variación de la ejecución del gesto siguiendo un protocolo de medición de ángulos en el plano sagital con uso de marcadores en punto anatómicos (Charmant, 2019). 
Las acciones de juego dependen, en gran medida, de las posibilidades individuales de los jugadores. Al respecto se puede ver que:

Cada deportista, teniendo en cuenta su individualidad, hace que sus movimientos dependan de la adaptación funcional a nuevos y diferentes esquemas restrictores que se presentan en el momento de la ejecución de un mismo patrón de movimiento y emergen de un comportamiento cooperativo de múltiples grados de libertad que posee su cuerpo y el ambiente externo. (Acero, 2009, p. 83)

Se utilizó el software Kinovea ${ }^{\circledR}$, una herramienta de fácil acceso utilizada por diferentes profesionales del deporte y la actividad física. "El editor de video Kinovea ${ }^{\circledR}$ es un programa de software libre y gratuito para el análisis de imágenes diseñado para el análisis del gesto y técnica deportiva para explorar y comentar una acción biomecánica” (Lluch, 2012, p. 1).

\section{Resultados}

En total, se realizaron 17 sesiones de entrenamiento del programa propuesto, una sesión de valoración pretest y una sesión de valoración postest. Para su elaboración se tuvo en cuenta la progresión de la carga física con respecto a la frecuencia, intensidad y volumen, teniendo en cuenta que:

Todo el concepto global del entrenamiento de flexibilidad puede sostenerse en la "Teoría del Estrés Físico" (Mueller y Maluf, 2002). En términos generales, esta teoría entiende que el ejercicio físico genera estrés físicomecánico (fuerza aplicada sobre un tejido biológico) al cual, el tejido biológico deberá adaptarse. (Medrano y Tortosa, 2012, p. 16)

El uso de la escala de esfuerzo percibido en la flexibilidad (Perflex) fue de gran aporte para la regulación progresiva de la intensidad de los estiramientos. Al respecto, Dantas y colaboradores (2008) destacan que:

Los resultados encontrados en esta investigación corroboran estudios encontrados en la literatura, que describen la PSE como un instrumento poderoso en la evaluación de la flexibilidad, pudiendo ser alterada por el entrenamiento, al provocar el aumento de los arcos articulares originales. (p. 294) 
Las sesiones de entrenamiento de la flexibilidad siempre fueron realizadas luego del calentamiento, teniendo en cuenta que según el metaanálisis de Dias (2009) "el estiramiento muscular, más la combinación de estiramiento más calentamiento resultaron ser los tratamientos más efectivos” (p. 10).

Los diferentes test, a excepción del análisis de gesto, no fueron ejecutados por la misma persona que diseñó el proyecto, esto con el fin de evitar el sesgo subjetivo y mantener la fiabilidad del estudio.

\section{Caracterización de la línea de base}

La población objeto de estudio inicial estaba conformada por 8 jugadores entre los 17 y 69 años con discapacidad física, de los cuales seis de ellos presentaban amputación total o parcial de miembro inferior (ninguno a nivel bilateral), un jugador con ausencia de mano izquierda (a causa de amelia) $\mathrm{y}$ un jugador con secuela de polio en miembros inferiores. El promedio de edad de los jugadores fue de 37.3 años y el promedio de tiempo de práctica en el voleibol sentado fue de 5.04 años. Se confirmó un promedio de edad elevado, así como un rango de edades variable; esto evidenció una similitud con el estudio realizado por Yüksel y Sevindi, (2018) en la selección turca de voleibol sentado.

En la tabla 2 se muestran los resultados iniciales sobre las diferentes pruebas aplicadas en el pretest, a excepción de la evaluación de la ejecución del golpe de antebrazos, considerando que los resultados del análisis de esta prueba deben relacionarse más adelante con el análisis del postest.

Tabla 2. Caracterización de línea de base

\begin{tabular}{ccccc}
\hline Test aplicados & Mínimo & Máximo & Media & Baremos \\
\hline Sit-and-reach pretest & -13.20 & 13.00 & 3.05 & Normal \\
$\begin{array}{c}\text { Shoulder neck } \\
\text { mobility pretest }\end{array}$ & 1.00 & 3.00 & 1.75 & $\begin{array}{c}\text { Moderada a severa } \\
\text { restricción }\end{array}$ \\
$\begin{array}{c}\text { Shoulder stretch } \\
\text { derecho pretest }\end{array}$ & 2.00 & 3.00 & 2.40 & Relativamente alto \\
$\begin{array}{c}\text { Shoulder stretch } \\
\text { izquierdo pretest }\end{array}$ & 1.00 & 3.00 & 1.80 & Bajo
\end{tabular}




\begin{tabular}{ccccc} 
Trunk lift pretest & 19.00 & 41.00 & 28.16 & Excelente \\
$\begin{array}{c}\text { Alcance funcional } \\
\text { frontal pretest }\end{array}$ & 38.00 & 47.00 & 41.00 & Normal \\
$\begin{array}{c}\text { Alcance funcional } \\
\text { derecho pretest }\end{array}$ & 12.00 & 30.00 & 21.68 & No se encuentra baremo \\
$\begin{array}{c}\text { Alcance funcional } \\
\text { izquierdo pretest }\end{array}$ & 17.50 & 29.00 & 23.08 & \\
\hline
\end{tabular}

Fuente: elaboración propia.

\section{Comparación pretest y postest}

Luego de la ejecución del programa de entrenamiento de la flexibilidad, se aplicó el postest. A la evaluación solo asistieron 6 jugadores de los 8 iniciales, ya que por motivos personales el jugador con secuela de polio no pudo asistir y tampoco el jugador con amputación de miembro inferior (unilateral). De tal manera, el análisis de los resultados del proyecto se hizo sobre la base de 6 jugadores, de los cuales 5 tenían amputación parcial o total de miembro inferior (unilateral) y un jugador tenía ausencia de la mano izquierda a causa de amelia. Para el análisis y comparación de los resultados se utilizó el software estadístico spss (tabla 3).

Tabla 3. Datos estadísticos descriptivos

\begin{tabular}{ccccc}
\hline & Mínimo & Máximo & Media & $\begin{array}{c}\text { Desviación } \\
\text { típica }\end{array}$ \\
\hline Asistencias & 4.00 & 15.00 & 9.8333 & 3.54495 \\
Sitandreachpre & -13.20 & 13.00 & 3.0500 & 10.81365 \\
Sitandreachpost & 1.50 & 14.50 & 9.6167 & 4.97289 \\
& & & & \\
Shoulderneckmobilitypre & 1.00 & 3.00 & 1.7500 & .98742 \\
Sholderneckmobilitypost & 2.50 & 4.00 & 3.2500 & .61237
\end{tabular}




\begin{tabular}{ccccc} 
Shoulderstretchderechopre & 2.00 & 3.00 & 2.4000 & .54772 \\
Shoulderstretchderechopost & 2.00 & 3.00 & 2.4000 & .54772 \\
Shoulderstretchisquierdopre & 1.00 & 3.00 & 1.8000 & .83666 \\
Shoulderstretchisquierdopost & 1.00 & 2.00 & 1.4000 & .54772 \\
Alcancefrontalpre & 38.00 & 47.00 & 41.0000 & 3.16228 \\
Alcancefrontalpost & 38.00 & 49.80 & 43.0000 & 4.46856 \\
Alcancederechopre & 12.00 & 30.00 & 21.6833 & 5.92635 \\
Alcancederechopost & 15.00 & 34.90 & 22.1500 & 6.93217 \\
Alcanceizquierdopre & 17.50 & 29.00 & 23.0833 & 3.85249 \\
Alcanceizquierdopost & 20.10 & 30.70 & $25, .333$ & 4.61288 \\
$\quad$ Tunkliftpre & 19.00 & 41.00 & 28.1667 & 7.75672 \\
Trunkliftpost & 23.50 & 42.00 & 31.0000 & 6.77495 \\
N válido (según lista) & & & & \\
\hline
\end{tabular}

Fuente: elaboración propia.

Según el análisis de los datos estadísticos descriptivos, las pruebas que mayor variación registraron al comparar el pretest y el postest fueron: el test sit-and-reach, el test shoulder neck mobility y el test trunk lift. Se observó una ligera variación en los resultados del test de alcance funcional y el de shoulder neck mobility. Luego de esto, se realizó la prueba de los rangos con signo de Wilcoxon para profundizar en el análisis de las variables (tabla 4). 
Tabla 4. Datos estadísticos de contraste

\begin{tabular}{|c|c|c|c|c|c|c|c|c|}
\hline & $\begin{array}{l}\text { sitandreach- } \\
\text { post - sitan- } \\
\text { dreachpre }\end{array}$ & $\begin{array}{c}\text { sholder- } \\
\text { neckmo- } \\
\text { bilitypost } \\
\text { - shoulder- } \\
\text { neckmobili- } \\
\text { typre }\end{array}$ & $\begin{array}{l}\text { shoulders- } \\
\text { tretchde- } \\
\text { rechopost } \\
\text { - shoulders- } \\
\text { tretchdere- } \\
\text { chopre }\end{array}$ & $\begin{array}{c}\text { shoulders- } \\
\text { tretchis- } \\
\text { quierdopost } \\
\text { - shoulders- } \\
\text { tretchisquier- } \\
\text { dopre }\end{array}$ & $\begin{array}{l}\text { alcance- } \\
\text { frontalpost } \\
\text { - alcance- } \\
\text { frontalpre }\end{array}$ & $\begin{array}{l}\text { alcancede- } \\
\text { rechopost } \\
\text { - alcancede- } \\
\text { rechopre }\end{array}$ & $\begin{array}{l}\text { alcanceiz- } \\
\text { quier- } \\
\text { dopost } \\
\text { - alcanceiz- } \\
\text { quierdopre }\end{array}$ & $\begin{array}{l}\text { trunkliftpost } \\
\text { - trunkliftpre }\end{array}$ \\
\hline Z & $-2.201(\mathrm{a})$ & $-2.251(\mathrm{a})$ & $.000(b)$ & $-1.000(\mathrm{c})$ & $-.944(a)$ & $-.943(a)$ & $-1.14(a)$ & $-2.201(a)$ \\
\hline $\begin{array}{l}\text { Significancia } \\
\text { asintótica } \\
\text { (bilateral) }\end{array}$ & .028 & .024 & 1.000 & .317 & .345 & .345 & .225 & .028 \\
\hline
\end{tabular}

Fuente: elaboración propia.

Como se puede observar, los test sit-and-reach, shoulder neck mobility y trunk lift presentaron significancia estadística. Aunque el test de alcance funcional no logró tener significancia estadística, sí se presentaron variaciones en los datos de la media del pretest y postest, por lo que puede decirse que tiene significancia clínica. Una vez realizado este análisis, se pasó a hacer el estudio de los resultados de la ejecución del golpe de antebrazos (tabla 5).

Tabla 5. Datos estadísticos descriptivos de la ejecución del golpe de antebrazos

\begin{tabular}{ccccc}
\hline & Media & $\begin{array}{c}\text { Desviación } \\
\text { típica }\end{array}$ & Mínimo & Máximo \\
\hline Agfxtrocopre & 53.5000 & 10.01499 & 41.00 & 69.00 \\
Agfxobropre & 107.8333 & 9.36839 & 97.00 & 124.00 \\
Agextrodillapre & 149.6667 & 9.00370 & 132.00 & 157.00 \\
& & & & \\
Agfxtrocopos & 48.0000 & 7.69415 & 42.00 & 63.00 \\
Agfxobropos & 112.8333 & 16.38800 & 81.00 & 127.00 \\
Agextrodillapos & 148.6667 & 18.34848 & 131.00 & 172.00 \\
\hline
\end{tabular}

Fuente: elaboración propia. 
Al respecto, se pueden observar pequeñas diferencias en la variación de la media estadística en los diferentes ángulos de flexión de tronco, las cuales no son estadísticamente significativas $(\mathrm{p}=0.141)$. Lo anterior permite afirmar que, con respecto al análisis de los pretest y postest de la ejecución del golpe de antebrazos, hay una ligera variación en los datos de la media, lo que representa una significancia a nivel clínico, pero no a nivel estadístico.

\section{Correlaciones}

Luego de emplear el coeficiente de correlación (Rho) de Spearman sobre las diferentes variables, se encontró una correlación significativa e inversamente proporcional $(\mathrm{r}=-0.88 ; \mathrm{p}=0.19)$ entre la flexión de tronco y los resultados de la prueba sit-and-reach, como se evidencia en estudios descritos previamente.

\section{Discusión}

Se evidenciaron cambios estadísticamente significativos en el desarrollo de la flexibilidad de la cadena muscular posterior y el hombro con respecto a los test sit-and-reach y shoulder neck mobility, de la misma manera que en el estudio de Coons y colaboradores (2017). Este estudio examinó el efecto del estiramiento estático y dinámico sobre los músculos isquiotibiales y concluyó que no hay diferencias significativas $(\mathrm{p}<0.001)$ entre los dos tipos de entrenamiento, ni en el rango de movilidad articular (range of motion [Rом]) posintervención.

Por otro lado, no se encontró significancia estadística en el test de alcance funcional ni en la ejecución del golpe de antebrazos, aunque se observaron cambios en los datos de la media en estos test, por lo cual puede decirse que hubo significancia clínica.

La significancia estadística en cuanto al desarrollo de la flexibilidad del tronco, hombro, así como el test trunk lift demostró el éxito del programa en este aspecto, así como las relaciones entre la flexibilidad de los músculos isquiotibiales y la flexión de tronco propuestas por Rodríguez (2014). Desde este punto de vista, la combinación de las técnicas FNP y dinámica, así como la estructura del programa, pueden ser tenidas en cuentas como 
modelo de entrenamiento de la flexibilidad. Esto en concordancia con el estudio de Sainz, Ayala y Cejudo (2012), en el que destaca que:

En cuanto a eficacia se refiere, no parece existir una técnica más eficaz que otra, por lo que todas (estática-activa, estática-pasiva, dinámica, FNP) parecen ser eficaces para la mejora del RoM articular y, por tanto, podrán ser utilizadas y combinadas como parte del entrenamiento de la flexibilidad. (p. 111)

Existe una correlación significativa entre el aumento del resultado en el test sit-and-reach y mayor inclinación del tronco hacia el frente en el golpe de antebrazos. Esto muestra que el entrenamiento de la flexibilidad de la cadena muscular posterior se debe tener en cuenta para facilitarle a los jugadores realizar la ejecución, no lo solo del golpe de antebrazos, sino de las diferentes acciones que requieren movimiento de inclinación del tronco en el voleibol sentado.

Estos hallazgos coinciden con los resultados del estudio de Manshouri, Rahnama y Khorzoghi (2014), quienes realizaron una investigación cuasiexperimental con población femenina universitaria que asistió a un curso de voleibol, para investigar los efectos del método Pilates sobre la flexibilidad y el servicio. Los resultados mostraron un efecto significativo en los efectos del pilates sobre el desarrollo de la flexibilidad con una $\mathrm{p}=0.05 \mathrm{de}$ significancia estadística y mejorando, de otra parte, la técnica del servicio.

Con el fin de lograr un mayor impacto sobre el alcance funcional, se debe tener en cuenta el componente de fuerza, ya que el desarrollo de la flexibilidad de forma individualizada no fue suficiente. Esto contrasta con el estudio de Wang, Onoda y Maruyama, (2017), el cual concluye que el entrenamiento FNP tiene efectos inmediatos sobre el test de alcance funcional en adultos hombres sanos. Así mismo, Méndez (2014) encontró en un grupo de personas mayores una mejora en el test de alcance funcional hacia delante, luego de un programa de actividad física que incluyó trabajo de flexibilidad y equilibrio.

Se recomienda la aplicación del programa de entrenamiento de la flexibilidad, teniendo en cuenta que el desarrollo de esta capacidad está directamente relacionada con la prevención de lesiones osteomusculares. De igual forma, para obtener mejores resultados se debe adicionar el componente de fuerza y agilidad en el diseño de programas de entrenamiento 
físico, como factores que influyen en el desempeño deportivo en el voleibol sentado (Jeoung, 2017) y el balance. Lo anterior teniendo cuenta que "la integridad física del atleta podría evitar no solo lesiones que puedan interferir en su desempeño, sino también contar con deportistas más longevos en competición" (García, Pujals, Fuster, Núñez y Rubio, 2014, citados por Morales, 2017. p. 4).

Por último, se recomienda trabajar los componentes de flexibilidad y movilidad de hombro de manera bilateral, ya que la caracterización de la línea de base muestra diferencias notorias en este aspecto. Al respecto, la batería ALPHA-Fit (Suni, Husu y Rinne, 2009) describe algunos de los test utilizados para medir estos componentes. Adicionalmente, es fundamental considerar las condiciones morfológicas de las articulaciones, lo que puede afectar directamente su movilidad (Zambrano y García, 2014).

\section{Referencias}

Acero, J. (2009). Aplicabilidad de la variabilidad en los análisis biomecánicos del gesto y el entrenamiento deportivo. En: Suárez, G. (ed.). Biomecánica deportiva y control del entrenamiento (pp. 45-85). Medellín: Funámbulos Editores.

Arias, O. (2011). Metodología de la investigación en las ciencias aplicadas al deporte: un enfoque cuantitativo. EFDeportes.com. Revista Digital, 16(157). Recuperado de https://www.efdeportes.com/efd157/investigacion-en-deporteenfoque-cuantitativo.htm

Busquet, L. (1994). Medicina deportiva. Las cadenas musculares: Tomo II. LordosisCifosis-Escoliosis y Deformaciones Torácicas. Tomo I. Tronco y Columna Vertebral. Barcelona: Paidotribo.

Castro, S. (2010). Unidad didáctica: iniciación al voleibol sentados. EfDeportes. com. Revista Digital, 14(142). Recuperado de https:/www.efdeportes.com/ efd142/iniciacion-al-voleibol-sentados.htm

Charmant, J. (2019). Kinovea (Version 0.8.15) [Windows mac]. Kinovea reproductor de video para análisis deportivo. Barcelona: Kinovea. Recuperado de https://www.kinovea.org/

Coons, J., Gould, C., Kim, J., Farley, R. y Caputo, J. (2017). Dynamic stretching is effective as static stretching at increasing flexibility. Journal of Human Sport and Exercise, 12(4), 1153-61. DoI: https://doi.org/10.14198/jhse.2017.124.02 
Dantas, M., Salomão, P., Gomes, d.S., Achour Júnior, A., Simão, R. y Figueiredo, A. (2008). Escala de Esfuerzo Percibido en la Flexibilidad (PERflex): ¿un instrumento adimensional para evaluarse la intensidad? Fitness \& Performance Journal, 7(5), 289-94. DOI: https://doi.org/10.3900/fpj.7.5.289.s

Fatih Yüksel, M. y Sevindi, T. (2018). Physical Fitness Profiles of Sitting Volleyball Players of the Turkish National Team. Universal Journal of Educational Research, 6(3), 556-561. DOI: 10.13189/ujer.2018.060318

Guerrero, Á. y Garnica, G. (2018). Efectos del entrenamiento de fuerza y flexibilidad en el alcance vertical en los jugadores de la selección Bogotá de Voleibol sentado (tesis de especialización), Corporación universitaria CENDA, Bogotá, Colombia.

Hernández, S., Fernández, C. y Baptista, L. (2010). Metodología de la investigación (5. ${ }^{\text {e ed. }) . ~ M e ́ x i c o: ~ M c G r a w-H i l l ~ I n t e r a m e r i c a n a . ~}$

Herrera, P., Ordóñez, P., Posada, T. y Landinez, P. (2014). Fisioterapia y balance en deportistas de voleibol sentado en la Selección Colombia de Voleibol Sentado rama masculina. Revista Cubana de Investigaciones Biomédicas [online], 33(4), 356-66. Recuperado de http://scielo.sld.cu/pdf/ibi/v33n4/ibi02414.pdf

Jeoung, B. (2017). Relationship between sitting volleyball performance and field fitness of sitting volleyball players in Korea. Journal of Exercise Rehabilitation, 13(6), 647-52. Dor: https://doi.org/10.12965/jer.1735170.585

Lluch, F. (2012). Análisis de imágenes: aplicación de KINOveA® en podología. El Peu, Revista de Podología, 33(2), 30-33. Recuperado de http://diposit.ub.edu/ dspace/bitstream/2445/122247/1/659242.pdf

Manshouri, M., Rahnama, N. y Khorzoghi, M. (2014). Effects of pilates exercises on flexibility and volleyball serve skill in female college students. Revista científica internacional de kinesiología, 11, 19-26. Recuperado de https://www. journalcra.com/article/effects-pilates-exercises-flexibility-and-volleyball-serveskill-female-college-students

Marszalek, J., Molik, B., Gomez, M., Skucas, K., Lencse-Mucha, J., Rekowski, W. y Kazmierska-Kowalewska, K. (2015). Relationships Between Anaerobic Performance, Field Tests and Game Performance of Sitting Volleyball Players. Journal of Human Kinetics, volume 48(1), 25-32. DOI: 10.1515/ hukin-2015-0088

Medrano, I. y Tortosa, L. (2012). La flexibilidad. Criterios básicos para su entrenamiento saludable aplicando la metodología pasivo-estática. Journal of Sport and Health Research, 4(1), 11-22. Recuperado de https:/dialnet.unirioja.es/ servlet/articulo?codigo $=4183364$ 
Méndez, S. (2014). Evaluación y Análisis de la Eficacia del Trabajo de la Flexibilidad de la Cadera Miofascial Recta posterior y del equilibrio sobre el Alcance funcional como predictor de caídas en personas mayores que realizan revitalización geriátrica (tesis doctoral). Universidad de Salamanca, Salamanca, España. DOI: https://doi.org/10.14201/gredos.125733

Morales, J. (2017). Voleibol sentado: comportamiento del brazo de apoyo en la ejecución técnica del remate (tesis de maestría). Universitad Miguel Hernández, Elche, España.

Morales, J. (2015). Protocolo de Valoración y Diagnóstico de la Condición Física y el Comportamiento Biomecánico para Practicantes de Voleibol Sentado (tesis de pregrado). Universidad Santo Tomás, Bogotá, Colombia. Recuperado de https://repository.usta.edu.co/handle/11634/4278

Mueller, M. y Maluf, K. (2002). Tissue Adaptation to Physical Stress: A Proposed "Physical Stress Theory" to Guide Physical Therapist Practice. Education, and Research. Physical Therapy, 82(4), 383-403. DoI: https://doi.org/10.1093/ ptj/82.4.383

Muñoz, G. y Montes, M. (2017). Voleibol sentado: un deporte que da sentido a la vida. Educación Física y Ciencia, 19(1), 019. DoI: https://doi. org/10.24215/23142561e019

Rodríguez Rodríguez, E. (2014). Rol de la musculatura isquiosural en el dolor bajo de la espalda: aumento de su extensibilidad como factor protector (tesis de pregrado). Universidad de Sevilla, Sevilla, España. Recuperado de https://idus. us.es/xmlui/handle/11441/32797

Ruiz, S. (2012). Deporte paralímpico: una mirada hacia el futuro. Revista U.D.C.A Actualidad \& Divulgación Científica, 15, 97-104. Recuperado de https://revistas.udca.edu.co/index.php/ruadc/article/view/897

Sainz, D., Ayala, F. y Cejudo, A. (2012). El entrenamiento de la flexibilidad: técnicas de estiramiento. Revista Andaluza de Medicina del Deporte, 5(3), 83-121. DOI: https://doi.org/10.1016/s1888-7546(12)70016-3

Sainz, D., Ayala, F., Cejudo, A. y Santonja, F. (2012). Descripción y análisis de la utilidad de las pruebas Sit-And-Reach para la Estimación de la flexibilidad de la Musculatura isquiosural. Revista Española de Educación Física y Deportes: REEFD, (396), 119-35. DoI: https://doi.org/10.1016/s1888-7546(12)70011-4

Sanz, M. (2014). Voleibol sentado, una alternativa para discapacitados (tesis de grado). Universidad Politécnica de Madrid, Madrid, España. 
Silva Dias, R. (2009). Eficacia de los tratamientos para la ganancia de flexibilidad en los musculos isquiotibiales. Metaanálisis (tesis doctoral). Universidad de Murcia, Murcia, España. Recuperado http://hdl.handle.net/10803/10871

Suni, J., Husu, P. y Rinne, M. (2009). Fitness for Health: The ALPHA-FIT Test Battery for Adults Aged 18-69 Tester's Manual. Finlandia-Europa del Norte: European Union, DG SANCO, and the UKK Institute for Health Promotion Research, Tampere, Finland.

Wang, H., Onoda, K. y Maruyama, H. (2017). Immediate effect of isometric resistance exercise on stretched hamstrings with proprioceptive neuromuscular facilitation on functional reach test of healthy male adults. International Meeting of Physical Therapy Science, 2(21), 61. Recuperado de https://spts.jpn.com/en/ meeting.html

World ParaVolley. (2017). Reglas oficiales de voleibol sentado 2017- 2020. World ParaVolley. Recuperado de http://paravolleypanam.com/communities/9/004/013/270/659/files/4635362739.pdf

Zambrano, L. y García, O. (2014). Métodos para el Desarrollo de la Flexibilidad en el Deporte: Ventajas y Desventajas de sus Técnicas de Entrenamiento (tesis de grado). Universidad del Valle, Santiago de Cali, Colombia. Recuperado de http:// bibliotecadigital.univalle.edu.co/bitstream/10893/7211/1/3484-0430890.pdf 
